\title{
ORIGINAL
}

\section{SEDENTARISMO, OBESIDAD Y SALUD MENTAL EN LA POBLACIÓN ESPAÑOLA DE 4 A 15 AÑOS DE EDAD}

\author{
Arturo Rodríguez-Hernández (1), Ernesto De la Cruz-Sánchez (2), Sebastián Feu (1) y \\ RaúlMartínez-Santos (3).
}

(1) Universidad de Extremadura

(2) Universidad de Murcia

(3) Universidad del País Vasco / Euskal Herriko Unibertsitatea

\section{RESUMEN}

Fundamento: Un estilo de vida activo y el mantenimiento de un peso saludable son dos de los principales determinantes del bienestar desde una perspectiva de salud pública. El objetivo del trabajo es evaluar el grado de asociación entre la actividad física y el índice de masa corporal (IMC) con el bienestar emocional y la salud mental de los escolares en España.

Método: Se evaluó el estado de salud mental a través del Cuestionario de Capacidades y Dificultades (SDQ), la práctica de actividad física durante el tiempo libre y el IMC en un total de 6.803 escolares de 4 a 15 años de edad, participantes en la Encuesta Nacional de Salud de España 2006. El grado de asociación entre variables fue estimado a través de un análisis de regresión logística multinomial.

Resultados: Entre los escolares sedentarios son más frecuentes los problemas de salud mental (OR 2,10), en concreto problemas emocionales $(\mathrm{OR} 1,84)$, los problemas de conducta (OR 1,53), los problemas con los compañeros (OR 2,35) y las dificultades de relación social (OR 1,36). Un índice de masa corporal (IMC) que denota obesidad se asocia a peor salud mental general (OR 1,58), mostrando los escolares obesos en mayor medida problemas emocionales (OR 1,52$)$ y problemas con compañeros (OR 2,43).

Conclusiones: En los escolares españoles un IMC saludable se asocia con mayor bienestar psicológico, aunque un estilo de vida activo es en mayor grado un buen indicador del estado de salud mental.

Palabras clave: Indice de masa corporal. Peso corpora 1.Estilo de vida sedentario. Calidad de vida. Infancia. España.

\section{ABSTRACT}

\section{Inactivity, Obesity and Mental Health in the Spanish Population from 4 to 15 Years of Age}

Background: An active lifestyle and a good weight status are two major health determinants from a public health perspective. Aim: To evaluate the degree of association between physical activity, weight status and the emotional and mental health of Spanish schoolchildren.

Method: Mental health status was assessed through the Strengths and Difficulties Questionnaire (SDQ), also leisure time physical activity and body mass index (BMI) in a total of 6803 children from 4 to 15 years participating in the Spanish National Health Survey 2006. The degree of association between. these variables was estimated by a multinomial logistic regression analysis.

Results: Among sedentary schoolchildren are more common mental health problems (OR 2.10), emotional problems (OR 1.84), conduct problems (OR 1.53), problems with peers (OR 2.35) and social relationship difficulties (OR 1.36). Obesity is associated with poor general mental health (OR 1.58), and obese schoolchildren show more often emotional problems (OR 1.52) and problems with peers (OR 2.43)

Conclusions: In the Spanish schoolchildren, a healthy BMI is associated with increased mental well-being, although an active lifestyle is the best indi ator of a good mental health status.

Key words: Body mass index. Body weight. Exercise. Sedentary life style. Quality of Life. Children.Spain

Ernesto De la Cruz-Sánchez

Universidad de Murcia (Campus de San Javier)

$\mathrm{C} /$ Argentina s/n,

30720 Santiago de la Ribera, España.

erneslacruz@um.es 


\section{INTRODUCCIÓN}

La elevada prevalencia de un estilo de vida sedentario en la población general, en la población infantil y juvenil en particular, supone un importante problema de salud pública $^{1}$. La prevalencia de un patrón insuficiente de actividad física oscila entre el $37 \%$ y el $40 \%$ en la población escolar española, en función de su sexo (niños y niñas, respectivamente) $)^{2}$. En la población general, la práctica regular de actividad física durante el tiempo libre se asocia de forma consistente a una mejor calidad de vida, mayor bienestar y el mantenimiento de una buena salud mental, siendo menos frecuente encontrar indicios de problemas psicológicos y psiquiátricos en aquellas personas que mantienen un estilo de vida activo $^{3}$.

La mayoría de los trabajos epidemiológicos que describen la asociación entre un estilo de vida activo y la salud mental se han llevado a cabo en población adulta ${ }^{3-5}$, aunque en los últimos años también se ha descrito la importancia del ejercicio físico regular $y$ el mantenimiento de un peso adecuado en las etapas en las que se conforma el estilo de $v i_{a}{ }^{6-8}$. Una persona sedentaria y con excesode peso en los primeros años de su vida tiene mayor probabilidad de mostrar estas mismas características en la edad adulta9, al igual que se ha descrito que la salud mental durante la infancia y la adolescencia determina la salud mental durante el resto de la vida ${ }^{10}$.

Desde una perspectiva integral del concepto de salud, la promoción de un estilo de vida activo puede contribuir a la mejora de la calidad de vida de las personas desde edades tempranas, ya que los escolares más activos declaran con mayor frecuencia una mejor y más estrecha relación con sus padres, mayor rendimiento académico, menores niveles de ansiedad y depresión, y conductas más saludables en lo que a hábitos nutricionales se refiere ${ }^{11-15}$. Tanto el ejercicio físico cotidiano como el mantenimiento de un peso corporal adecuado son también elementos potencialmente importantes para evitar la aparición temprana de comportamientos antisociales, el riesgo de exclusión social ${ }^{15-}$ ${ }^{17}$ y la adopción de conductas perjudiciales como el tabaquismo o el consumo de alcohol, asociadas de la misma forma a una mala salud física, mental y a un estilo de vida sedentario $^{18,19}$.

El objetivo de este trabajo es estimar el grado de asociación entre la práctica de actividad física durante el tiempo libre y el mantenimiento de un peso adecuado con el bienestar emocional y la salud mental de la población española de 4 a 15 años de edad.

\section{SUJETOS Y MÉTODO}

Diseño del estudio. Los datos empleados proceden de la Encuesta Nacional de Salud Infantil de España 2006 (ENSE 2006) ${ }^{20}$, estudio transversal desarrollado por el Ministerio de Sanidad y Política Social en el que se evalúa la salud de un total de 9.122 personas menores de 16 años, representativas del total de la población española de ese grupo de edad. La muestra se dividió en 50 submuestras provinciales, empleando un muestreo polietápico y estratificado por conglomerados en el que se tuvo en cuenta, en primer lugar, las secciones censales, en segundo los hogares $\mathrm{y}$ finalmente un individuo de cada hogar. Las secciones censales fueron elegidas dentro de cada estrato con probabilidad proporcional a su tamaño, mientras que los hogares e individuos fueron seleccionados de manera aleatoria teniendo en cuenta cuotas de edad y sexo (establecidas a partir del último censo oficial disponible).

Para minimizar sesgos estacionales en términos de morbilidad y estilo de vida, la entrevista se condujo en cuatro etapas de 15 
días, y el período de referencia de cadavariable explorada estaba compuesto por 2 semanas y 1 año desde el día de la recogida de datos. $\mathrm{La}$ recogida de información se llevó a cabo por entrevistadores previamente formados para la tarea.

Variables. La ENSE Infantil 2006 contiene por primera vez la versión española del Cuestionario de Capacidades y Dificultades (Strength and Difficulties Questionnaire, SDQ) en su versión para padres $^{21}$, un trabajo previamente validado y empleado en nuestro país ${ }^{22-24}$. La puntuación global de la salud mental es la suma de los resultados de las cinco subescalas que componen este cuestionario, que evalúan los problemas emocionales, los problemas de conducta, la hiperactividad, los problemas con los compañeros y las dificultades de relación social. Los puntos de corte originalmente propuestos para establecer la normalidad en los resultados de esta prueba han sido utilizados como referencia en el presente trabajo ${ }^{21}$.

Para la estimación del patrón de actividad física habitual, los padres o personas responsables de los niños respondieron a la siguiente pregunta: ¿cuál de estas posibilidades describe mejor la frecuencia con la que el/la niño/a realiza alguna actividad física en su tiempo libre? para la que había cuatro respuestas opcionales, a partir de las cuales se clasificó a los sujetos $^{14}$ : sedentarismo, practica ocasional, practica regular-mensual y participación semanal en actividades físicas y deportivas.

Para obtener el índice de masa corporal (IMC), definido como el peso en kilos dividido por la talla en metros al cuadrado, se emplearon el peso y la talla de los niños autodeclarados por los padres o la persona responsable, según las siguientes preguntas: ¿podría decirme cuánto pesa el/la niño/a aproximadamente, sin zapatos ni ropa? ¿Y cuánto mide, aproximadamente, sin zapatos? Una vez obtenido el IMC se utilizaron los criterios de Cole et $\mathrm{al}^{25}$ para la clasificación según el estatus peso. El procedimiento para obtener estos datos en la ENSE 2006 constituye un método ya validado en este tipo de estudios con población en edad escolar ${ }^{26,27}$.

La clase social se estableció a partir de la propuesta de la Sociedad Española de Epidemiología, basada en la clasificación de Goldthorpe y asignada a cada unidad familiar de acuerdo con la ocupación del sustentador principal del hogar ${ }^{28}$.

Análisis estadístico. Las estimaciones fueron ponderadas para tener en cuenta el diseño muestral. Posteriormente se implementó un modelo de regresión logística multinomial, calculándose las odds ratio y sus respectivos intervalos de confianza al $95 \%$ para establecer el grado de asociación entre las variables que fueron objeto de estudio. En los análisis realizados se comparan, en primer lugar, los sujetos sedentarios con el resto, y de la misma forma los que son clasificados como obesos respecto a los que no lo son para proceder posteriormente a un análisis más detallado en el que se contemplan los cuatro niveles de actividad física y los tres niveles de IMC. Para el análisis se utilizó el programa SPSS, versión 19.0.

\section{RESULTADOS}

En tabla 1 se describen las características de la muestra. Tal y como puede apreciarse en la figura 1, al comparar a los sujetos sedentarios con el resto se constató que era más frecuente observar en ellos problemas emocionales $(\mathrm{OR}=1,84)$, problemas de conducta $(\mathrm{OR}=1,53)$, problemas con los compañeros $(\mathrm{OR}=2,35)$ y dificultades de relación social $(\mathrm{OR}=1,36)$. No realizar actividad física durante el tiempo libre supuso una mayor prevalencia de problemas de salud mental $(\mathrm{OR}=2,10$ para la suma total de dificultades), de forma que un estilo 
Tabla 1

Prevalencia de problemas de salud mental estimados a través del SDQ (limite y anormal) en la muestra objeto de análisis en función de las variables estudiadas

\begin{tabular}{|c|c|c|c|c|c|c|c|}
\hline & $\mathrm{n}$ & $\begin{array}{l}\text { Problemas } \\
\text { emocionales }\end{array}$ & $\begin{array}{c}\text { Problemas } \\
\text { de } \\
\text { conducta }\end{array}$ & Hiperactividad & $\begin{array}{c}\text { Problemas } \\
\text { con los } \\
\text { conpañeros }\end{array}$ & Prosocial & $\begin{array}{c}\text { Total } \\
\text { dificultades }\end{array}$ \\
\hline Total & 6803 & $19,7 \%$ & $30 \%$ & $31 \%$ & $16,8 \%$ & $6,9 \%$ & $20,8 \%$ \\
\hline \multicolumn{8}{|l|}{ Sexo } \\
\hline Niños & $3491(51,3 \%)$ & $19,3 \%$ & $32,7 \%$ & $36,9 \%$ & $18,5 \%$ & $7,9 \%$ & $23,2 \%$ \\
\hline Niñas & $3312(48,7 \%)$ & $22,2 \%$ & $30,4 \%$ & $28,2 \%$ & $16,8 \%$ & $6,7 \%$ & $21 \%$ \\
\hline \multicolumn{8}{|l|}{ Grupo de edad } \\
\hline $4-6$ & $1585(23,3 \%)$ & $16,7 \%$ & $37,7 \%$ & $38,8 \%$ & $15,5 \%$ & $10,3 \%$ & $23 \%$ \\
\hline $7-9$ & $1678(24,7 \%)$ & $24,5 \%$ & $34,3 \%$ & $36,5 \%$ & $19,3 \%$ & $5,7 \%$ & $26,2 \%$ \\
\hline $10-12$ & $1730(25,4 \%)$ & $22 \%$ & $28 \%$ & $28,7 \%$ & $18 \%$ & $6,4 \%$ & $21 \%$ \\
\hline $13-15$ & $1810(26,6)$ & $19,6 \%$ & $27,2 \%$ & $27,5 \%$ & $17,9 \%$ & $6,9 \%$ & $18,6 \%$ \\
\hline \multicolumn{8}{|l|}{ Clase social } \\
\hline I-II & $1583(23,3 \%)$ & $15,6 \%$ & $24 \%$ & $21,4 \%$ & $13,1 \%$ & $5,5 \%$ & $14,2 \%$ \\
\hline III & $1604(23,6 \%)$ & $19 \%$ & $28,1 \%$ & $31 \%$ & $15,4 \%$ & $7,3 \%$ & $18,2 \%$ \\
\hline IV-V & $3511(51,6 \%)$ & $23,8 \%$ & $36,6 \%$ & $38,8 \%$ & $20,7 \%$ & $8,1 \%$ & $27,5 \%$ \\
\hline Perdidos & $105(1,5 \%)$ & $17 \%$ & $21,2 \%$ & $13,5 \%$ & $15,2 \%$ & $3,1 \%$ & $12,6 \%$ \\
\hline \multicolumn{8}{|l|}{ IMC } \\
\hline Normal & $3748(55,1 \%)$ & $19,4 \%$ & $30,2 \%$ & $31 \%$ & $16,6 \%$ & $6,5 \%$ & $20,4 \%$ \\
\hline Sobrepeso & $1022(15 \%)$ & $20,9 \%$ & $29,9 \%$ & $28,2 \%$ & $15,3 \%$ & $7,5 \%$ & $19 \%$ \\
\hline Obesidad & $501(7,4 \%)$ & $27,8 \%$ & $38,3 \%$ & $38,4 \%$ & $32 \%$ & $7,9 \%$ & $31,2 \%$ \\
\hline Perdidos & $1532(22,5 \%)$ & $20,2 \%$ & $32 \%$ & $35,5 \%$ & $16,3 \%$ & $8,2 \%$ & $23,7 \%$ \\
\hline \multicolumn{8}{|c|}{ Patrón de actividad física } \\
\hline No practica & $745(10,9 \%)$ & $31,6 \%$ & $40,5 \%$ & $39,1 \%$ & $30,7 \%$ & $9,2 \%$ & $35,4 \%$ \\
\hline Ocasional & $2570(37,8)$ & $21,8 \%$ & $33,2 \%$ & $34,8 \%$ & $18,6 \%$ & $9,7 \%$ & $23,6 \%$ \\
\hline Regular/mes & $1790(26,3 \%)$ & $18,3 \%$ & $28,4 \%$ & $28,9 \%$ & $15,2 \%$ & $6,8 \%$ & $18,6 \%$ \\
\hline Regular/semana & $\begin{array}{c}1557 \\
(22,9 \%)\end{array}$ & $16,8 \%$ & $28,2 \%$ & $30,4 \%$ & $12,3 \%$ & $3 \%$ & $17,2 \%$ \\
\hline Perdidos & $142(2,1 \%)$ & $4,1 \%$ & $12,2 \%$ & $11,3 \%$ & $10,6 \%$ & $1,8 \%$ & $9,5 \%$ \\
\hline
\end{tabular}

de vida activo en alguna medida se asocia de forma significativa a una mejor salud mental en el total de la muestra.

Un IMC por encima del punto de corte que delimita la clasificación de obesidad empleada en este trabajo se asoció a peores resultados globales obtenidos en el SDQ (figura 2). Comparados con sus compañeros, los sujetos obesos mostraron 1,58 veces más problemas de salud mental global para el total de las subescalas. Los que tenían un IMC igual o mayor a 30 mostraron con más frecuencia problemas emocionales $(\mathrm{OR}=1,52)$ y problemas de relación con los compañeros $(\mathrm{OR}=2,43)$.

El análisis detallado de los hallazgos en función de las diferentes subescalas permitió describir que la asociación de la salud mental con el patrón de actividad física habitual era creciente, a medida que el patrón de actividad física es mayor los sujetos tuvieron mejor salud mental (tabla 2).

En el grupo sedentario se observaron diferencias estadísticamente significativas $(\mathrm{p}<0,05)$ para todos los valores anormales y límites en todas las subescalas (exceptuando los valores límite en las subescalas de problemas de conducta e hiperactividad). Subiendo un nivel, la práctica ocasional se asoció también en mayor medida a problemas de salud mental en todas las subescalas, excepto en la que evaluó problemas de conducta $(\mathrm{OR}=1,16)$. Sin embargo, en el nivel más próximo a un 


\section{Figura 1}

Asociación entre un estilo de vida sedentario y la prevalencia de problemas de salud mental (suma de límite más anormal) en las diferentes escalas del cuestionario SDQ. OR ajustado a sexo, edad y clase social

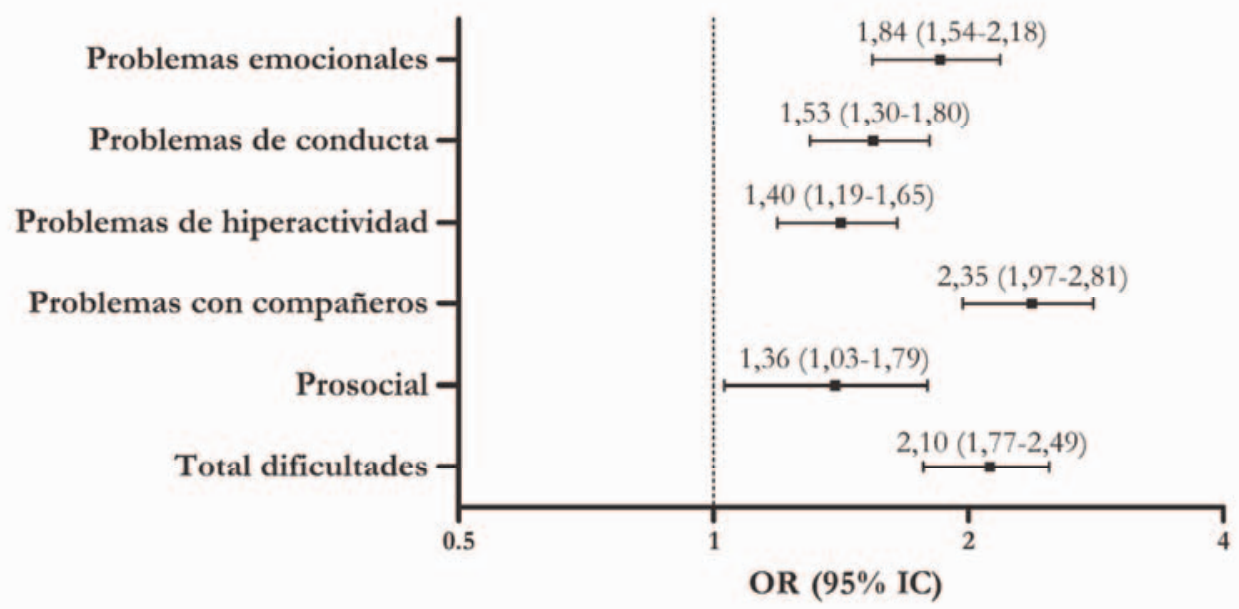

Figura 2

Asociación entre obesidad y la prevalencia de problemas de salud mental (suma de límite más anormal) en las diferentes escalas del cuestionario SDQ. OR ajustado por sexo, edad y clase social

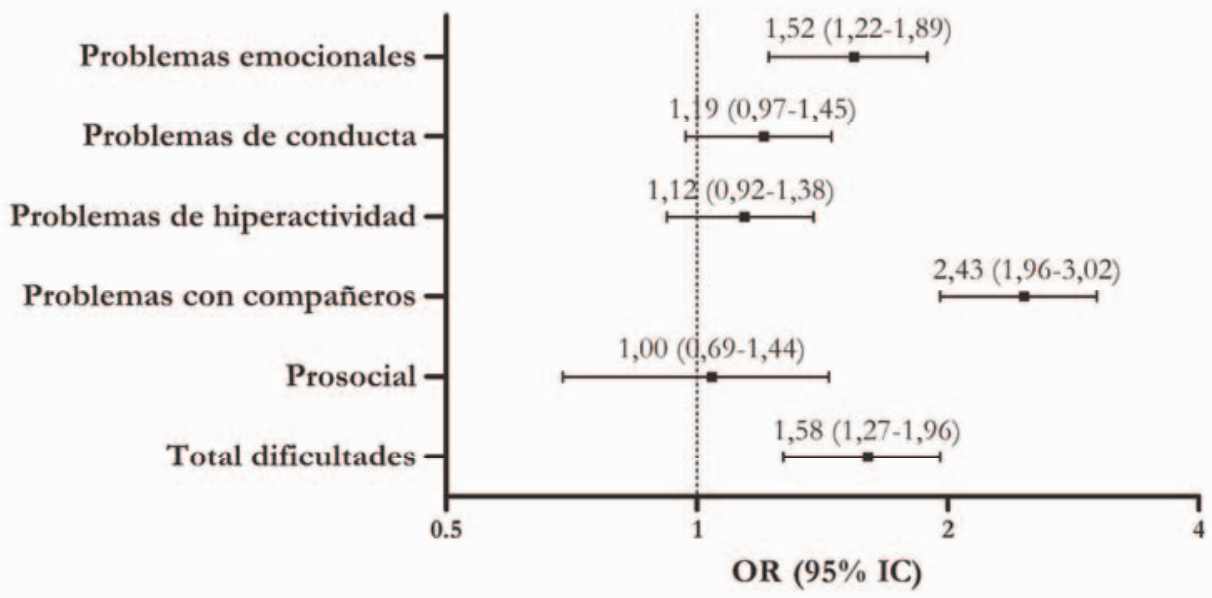




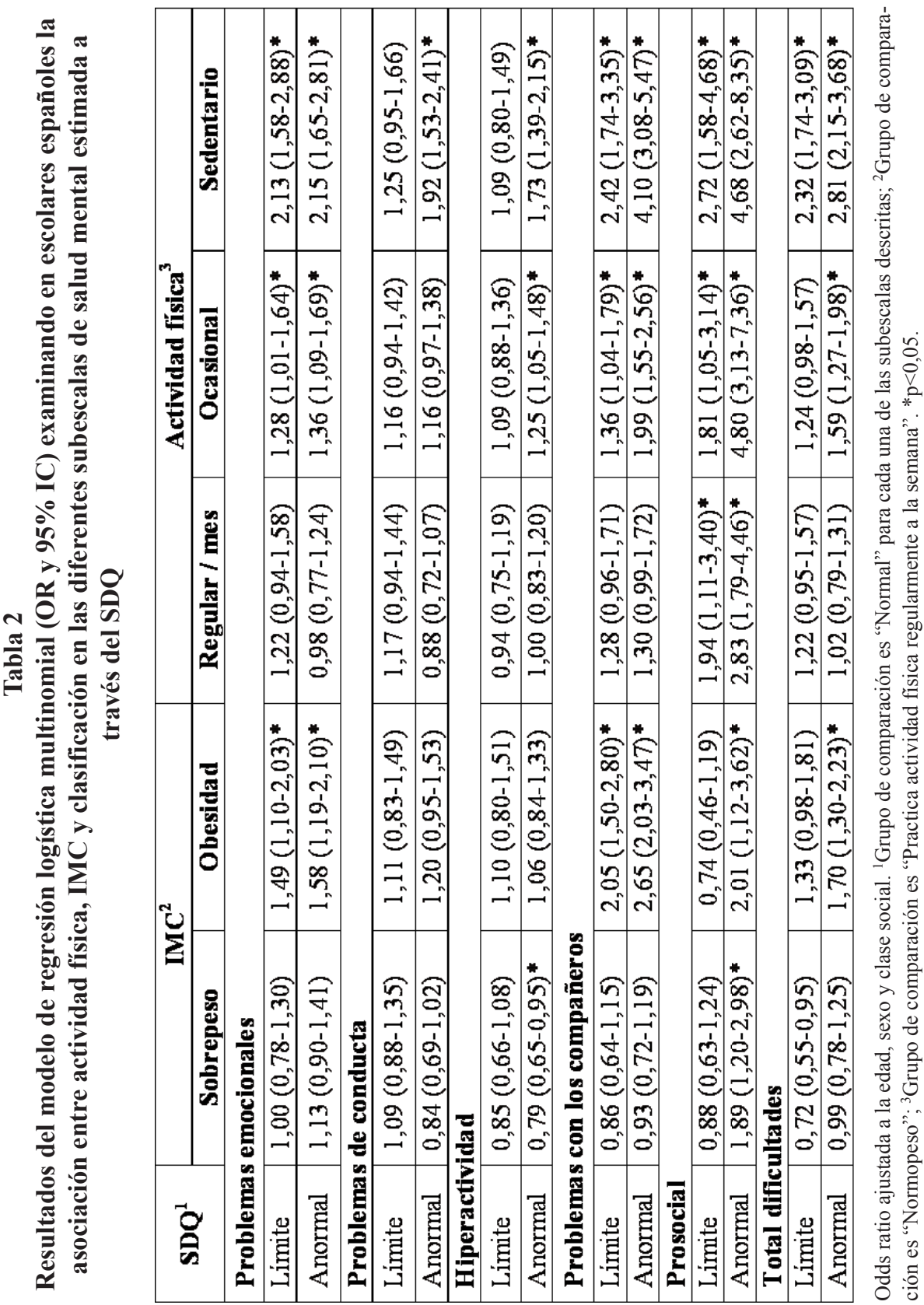


estilo de vida activo, práctica regular, sólo fue más frecuente observar diferencias en la puntuación de las subescala prosocial $(1,94$ para límite y 2,83 para anormal respecto a los que realizaban actividad física durante toda la semana).

Cabe destacar el caso de la subescala prosocial, en la que existen grandes diferencias en todos los grupos respecto a los sujetos activos (OR's de 1,94-1,81-2,72 para clasificación en grupo límite y OR's 2,83-4,80-4,68 para ser clasificado en grupo anormal, respectivamente para cada grupo analizado regular-ocasional-sedentario). Odds ratio ajustada a la edad, sexo y clase social. 1 Grupo de comparación es "Normal" para cada una de las subescalas descritas; 2 Grupo de comparación es "Normopeso"; 3 Grupo de comparación es "Practica actividad física regularmente a la semana", * $\mathrm{p}<0,05$.

La obesidad se asoció significativamente al conjunto de problemas en la categoría anormal (OR's 1,70), a los problemas emocionales (OR's de 1,49 y 1,58 para límite y anormal, respectivamente), a problemas con compañeros (OR's de 2,05 y 2,65 para límite y anormal) y prosocial (OR 2,01 para anormal). En el resto de las dimensiones no se mostraron asociaciones estadísticamente significativas. Por lo que respecta al sobrepeso, se asoció solamente a problemas en la escala prosocial $(\mathrm{OR}=1,89$ para anormal).

\section{DISCUSIÓN}

El presente trabajo permite describir que la obesidad y el sedentarismo son factores que se asocian en gran medida a un peor estado de salud mental en la población estudiada. A nivel global, el $20,8 \%$ de los niños y niñas estudiados padece algún tipo de trastorno mental que afecta a su vida cotidiana, a la de sus pares, familiares y educadores. Este fenómeno ha sido ya descrito como un problema que persiste en nuestro país y se relaciona con otros problemas emergentes, como son los profundos cambios en el estilo de vida ${ }^{29}$.

En el caso de la obesidad, establecida a través de la valoración del IMC, se puede describir que un peso saludable se asociacon el bienestar psicológico en la población estudiada, tal como se observa al comparar a los sujetos obesos con el resto. Otros trabajos han definido esta misma relación en poblaciones similares a la analizada aquí, concluyendo además que la obesidad es un estigma social que dificulta las relaciones personales ${ }^{30-34}$. Según estos autores, la relación entre obesidad y salud mental es causa y efecto de dificultades de relación personal: los escolares obesos tienen más dificultades en sus relaciones personales y tener dificultades en estas relaciones se socia a incrementos de peso poco saludables ${ }^{34}$.

La mayor prevalencia de trastornos emocionales en los escolares obesos que hemos observado en este estudio ha sido objeto de análisis en estudios previos, describiendo también algunos autores que el exceso de peso y una mala imagen corporal se asocian a la aparición de síntomas depresivos $^{35}$. Un estilo de vida activo se asocia a mejor salud mental $\mathrm{y}$, aún no observándose en algunos casos significación estadística, existe una tendencia generalizada de asociación entre el patrón de ctividad física durante el tiempo libre y la salud mental. En la valoración global obtenida con el SDQ (total dificultades) las odds ratio de las puntuaciones límite o anormales disminuyen a medida que se mantiene un estilo de vida más activo. Esta asociación ha sido establecida en otros países en trabajos con grandes muestras, si bien con otros instrumentos e indicadores propuestos para valorar la salud mental, distintos al cuestionario $\mathrm{SDQ}^{36-38}$. No obstante, las dimensiones que proporciona el cribado del SDQ mantienen una correspondencia 
directa con las de la salud mental de los trabajos citados, por lo que los hallazgos aquí descritos concuerdan con las principales conclusiones de los estudios referidos.

Acorde con los resultados de este trabajo, en los que destaca la asociación entre sedentarismo y problemas de relación social $\mathrm{y}$ con los compañeros, se ha descrito previamente que la inactividad física perjudica la socialización en la población infantil ${ }^{15}$. De la misma forma $y$ en correspondencia con este estudio, la inactividad física también conlleva una mayor prevalencia de problemas de conducta $^{16}$.

Los resultados de este estudio de corte transversal concuerdan con otros trabajos con un diseño muy distinto y en diferentes contextos: en menor escala, se ha evaluado la relación ejercicio físico-salud mental través de la implementación de programas de actividad física dirigida en grupos reducidos, encontrando que existe asociación entre ambos factores, y además causal (algo que no podemos inferir del presente trabajo ${ }^{39}$. Los resultados del presente trabajo pueden atribuirse en parte a las adaptaciones derivadas de un estilo de vida activo, que reducen la incidencia de problemas de salud mental, depresión o ansiedad $^{40}$

Es necesario apuntar que las herramientas de evaluación incluidas en la ENSE Infantil de 2006 no están exentas de limitaciones: en primer lugar, el SDQ no permite elaborar un diagnóstico psiquiátrico clínico, aunque es un instrumento eficiente para estimar el estado de salud mental infantil en grandes muestras, y además se ha constatado como válido y fiable en nuestro país ${ }^{22-25}$. En segundo lugar, la estimación del patrón de actividad física no es tan precisa como la que pueden ofrecer otro tipo de metodologías para estimar estas variables (por ejemplo el empleo de instrumentos de registro mecánicos para valorar el gasto energético), pero es aceptada y empleada en otros trabajos ${ }^{15,27}$. En tercer lugar, la pérdida de valores del IMC en la clasificación del estatus de peso de lossujetos mediante este procedimiento (el 22,5\% de los sujetos de esa edad que conforman la muestra original de la ENSE 2006) podría introducir algunos sesgos: el reporte del peso de los hijos por parte de sus padres podría estar condicionado por variables como el propio peso de los padres, su clase social o lo que socialmente puede ser aceptado como un peso normal ${ }^{41}$. No obstante, la dirección de ese sesgo en la ENSE 2006 ya ha sido descrita y permite estimar la prevalencia de exceso de peso y obesidad desde un punto de vista conservador, existiendo evidencias de que la prevalencia de exceso de peso real es al menos tan alta como la descrita en este estudio $^{41}$.

Este trabajo es uno de los primeros de su tipo en nuestro país, realizado en una amplia base poblacional y con el objetivo de definir la asociación entre salud mental, actividad física y obesidad. Desde una perspectiva de salud pública, permite describir que las consecuencias en términos de salud mental del sedentarismo y la obesidad se observan en las primeras etapas de la vida, y en la población escolar española (entre los 4 y 15 años de edad) un peso saludable se asocia con diferentes dimensiones del bienestar psicológico, mientras que la práctica de actividad física durante el tiempo libre es un buen indicador del estado de la salud infantil en el más amplio de sus sentidos.

\section{BIBLIOGRAFÍA}

1. Blair SN. Physical inactivity: the biggest publichealth problem of the 21st century. Br J Sports Med.2009; 43:1-2.

2. Roman B, Serra-Majem L, Ribas-Barba L, PérezRodrigo C, Aranceta J. How many children and adolescents in Spain comply with the recommendations on physical activity? J Sports MedPhys Fitness. 2008; 48(3):380-7. 
3. De la Cruz-Sanchez E, Moreno-Contreras MI, PinoOrtega J, Martínez-Santos R. Leisure time physical activity and its relationships with some mental health indicators in Spain through the National Health Survey. Salud Ment. 2011; 34(1):45-52.

4. Abu-Omar K. Mental health and physical activity in the European Union. Soz Praventivmed. 2004; 49(5):301-9.

5. Goodwin RD. Association between physical activity and mental disorders among adults in the United States. Prev Med. 2003; 36:698-703.

6. Ussher MH, Owen CG, Cook DG, Whincup PH. The relationship between physical activity, sedentary behavior and psychological well-being among adolescents. Soc Psych Psych Epid. 2007; 42:851-6.

7. Sagatun A, Sogaard AJ, Bjertness E, Selmer R, Heyerdahl S. The association between weekly hours of physical activity and mental health: a three-year follow-up study of 15-16-year-old students in the city of Oslo, Norway. BMC Public Health. 2007; 7:155.

8. Sacker A, Cable N. Do adolescent leisure-time physical activities foster health and well-being in adulthood? Evidence from two British birth cohorts. Eur J Public Health. 2006; 16(3):332-6.

9. Ong KK. Early determinants of obesity. Endocr Dev. 2010; 19:53-61.

10. Goodman A, Joyce R, Smith JP. The long shadow cast by childhood physical and mental problems on adult life. Proc Natl Acad Sci U S A. 2011; 108(15):6032-7.

11. Steptoe A, Butler N. Sports participation and emotional wellbeing in adolescents. Lancet. 1996; 347:1789-92.

12. Norris R, Carrol D, Cochrane R. The effects of physical activity and exercise training on psychological stress and well-being in an adolescent population. J Psychosomat Res. 1992; 36:55-65.

13. Brosnahan J, Steffen LM, Lytle L, Patterson J, Boostrom $\mathrm{A}$. The relation between physical activity and mental health among Hispanic and non-Hispanic white adolescents. Arch Pediatr Adolesc Med. 2004; 158:818-23.

14. Lasheras L, Aznar S, Merino B, Gil López E. Factors associated with physical activity among Spanish youth through the National Health Survey. Prev Med. 2001; $32: 455-64$

15. Field T, Diego M, Sanders CE. Exercise inpositively related to adolescents' relationships and academics. Adolescence. 2001; 36:105-10.
16. Wiles NJ, Jones GT, Haase AM, Lawlor DA, Macfarlane GJ, Lewis G. Physical activity and emotional problems amongst adolescents. A longitudinal study. Soc Psych Psych Epid. 2008; 43(10):765-72.

17. King KA, Tergerson JL, Wilson BR. Effect of social support on adolescents' perceptions of and engagement in physical activity. J Phys Act Health. 2008; 5(3):374-84.

18. Kantomaa MT, Tammelin TH, Ebeling HE, Taanila AM. Emotional and behavioral problems in relation to physical activity in youth. Med Sci Sports Exerc. 2008; 40:1749-56

19. Clark C, Haines MM, Head J, Klineberg E, Arephin M, Viner R, et al. Psychological symptoms and physical health and health behaviours in adolescents: a prospective 2-year study in East London. Addiction. 2006; 102:12635 .

20.- Ministerio de Sanidad y Política Social. Encuesta Nacional de Salud 2006. Disponible en: http:/www.msc.es/estadEstudios/estadisticas/estadisticas/microdatos/frmListadoMicrodatos.jsp.

21. Goodman R. Psychometric properties of the strengths and difficulties questionnaire. J Am Acad Child Adolesc Psychiatr. 2001; 40:1337-45.

22. Rajmil L, López-Aguilà S, Mompart Penina A, Medina Bustos A, Rodríguez Sanz M, Brugulat Guiteras P. Socio-economic inequalities in children's mental health in Catalonia. An Pediatr. 2010;73(5):233-40.

23. Rajmil L, Palacio-Vieira JA, Herdman M, LópezAguilà S, Villalonga-Olives E, Valderas JM, et al.Effect on health-related quality of life of changes in mental health in children and adolescents. Health Qual Life Outcomes. 2009; 7:103

24. Marzocchi GM, Capron C, Di Pietro M, Duran Tauleria E, Duyme $M$, Frigerio A, et al. The use of the Strengths and Difficulties Questionnaire (SDQ) in Southern European countries. Eur Child Adolesc Psychiatry. 2004;13 (Suppl 2):1140-6.

25. Cole TJ, Flegal KM, Nicholls D, Jackson A. Establishing a standard definition for child overweight and obesity worldwide: international survey. BMJ. 2000; 320(7244):1240-3

26. Burdette HL, Whitaker RC, Daniels SR. Parentalreport of outdoor playtime as a measure of physical activity in preschool-aged children. Arch Pediatr Adolesc Med. 2004; 158(4):353-7.

27. Garcia-Marcos L, Valverde-Molina J, Sanchez-Solis M, Soriano-Pérez MJ, Baeza-Alcaraz A, Martinez-Torres 
A et al. Validity of parent-reported height and weight for defining obesity among asthmatic and nonasthmatic schoolchildren. Int Arch Allergy Immunol. 2006; 139:139-145.

28. Regidor E. The Goldthorpe Social Class Classification: reference framework for a proposal for the measurement of social class by the Working Group of the Spanish Society of Epidemiology. Rev Esp Salud Publica. 2001; 75(1):13-22.

29. Colomer-Revuelta C, Colomer-Revuelta J, Mercer R, Peiró-Pérez R, Rajmil L. Child health. Gac Sanit. 2004;18 (Suppl 1):39-46.

30. Latner JD, Stunkard AJ. Getting worse: the stigmatization of obese children. Obes Res. 2003; 11: 452-6.

31. Swallen KC, Reither EN, Haas SA, Meier AM. Overweight, obesity, and health-related quality of life among adolescents: the National Longitudinal Study of Adolescent Health. Pediatrics. 2005; 115(2):340-7.

32. Eisenberg ME, Neumark-Sztainer D, Story M. Associations of Weight-Based Teasing and Emotional Well-being Among Adolescents. Arch Pediatr Adolesc Med. 2003; 157: 733-8.

33. Midei AJ, Matthews KA. Interpersonal violence in childhood as a risk factor for obesity: a systematic review of the literature and proposed pathways. Obes Rev. 2011; 12: e159-e172.

34. Adams RE, Bukowski WM. Peer victimization as a predictor of depression and body mass index in obese and non-obese adolescents. J Child Psychol Psychiatry. 2008; 49: 858-66.

35. Siegel JM. Body image change and adolescent depressive symptoms. J Adolesc Res. 2002; 17(1):27- 41.

36. Sanders C. Moderate involvement in sports is related to lower depression levels among adolescents. Adolescence. 2000; 35:793-7.

37. De Moor M, Beem A, Stubbe J, Boomsma D, De Geus E. Regular exercise, anxiety, depression and personality: a population-based study. Prev Med. 2006; 42:273-9.

38. Rothon C, Edwards P, Bhui K, Viner RM, Taylor S, Stansfeld SA. Physical activity and depressive symptoms in adolescents: a prospective study. BMC Med. 2010; $8: 32$.

39. Merry S, McDowell H, Hetrick S, Bir J, Muller N. Psychological and/or educational interventions for the prevention of depression in children and adolescents. Cochrane Database Syst Rev. 2004; 1:CD003380.
40. Larun L, Nordheim LV, Ekeland E, Hagen KB, Heian F. Exercise in prevention and treatment of anxiety and depression among children and young people. Cochrane Database Syst Rev. 2006; 3:CD004691.

41. Salcedo V, Gutierrez-Fisac JL, Guallar-Castillon P, Rodriguez-Artalejo F. Trends in overweight and misperceived overweight in Spain from 1987 to 2007. Int J Obes. (Lond). 2010, 34(12):1759-65. 